\title{
Formation of massive binaries
}

\author{
Hans Zinnecker \\ Astrophysikalisches Institut Potsdam, \\ An der Sternwarte 16, D-14482 Potsdam, BRD
}

\begin{abstract}
The formation of massive stars is one of the major unsolved problems in stellar astrophysics. However, only few if any of these are found as single stars, on average massive stars have more than one companion. Many of them are born in dense stellar clusters and several clusters have an excess of massive short-period spectroscopic binaries, with severe implication for binary-related stellar evolution including mergers, and also for the origin of massive runaway stars. The multiplicity of massive stars seems to increase with increasing primary mass and with increasing density of young star clusters. These observations suggest that massive binary and multiple systems originate mainly from dynamical gravitational interactions and accretion-induced protostellar collisions in dense clusters. If true, the binary properties of massive stars in less dense OB associations should be less extreme. This prediction should be tested by future observations. The paper reviews both the latest observations and theoretical ideas related to the origin of massive binaries. It concludes with a speculation on how the binary properties might change with metallicity (e.g., LMC/SMC).
\end{abstract}

\section{Introduction}

Most massive stars, if not all, are (or were) members of binary and multiple systems. Ignoring the multiplicity of massive stars can introduce severe problems when comparing observations and stellar evolution theory. For example, the stellar luminosity in the range $20-60 \mathrm{M}_{\odot}$ scales with the third power of the mass, and the rate of production of Lyman continuum photons scales with the fourth power of the mass. This means that a $50 \mathrm{M}_{\odot}$ star has four times the luminosity of an (unresolved) pair of two $25 \mathrm{M}_{\odot}$ stars, and eight times the amount of Lyman continuum photons per sec. This example, drastic as it may be, shows the importance of recognizing how the mass of a system is distributed among its components.

The basic properties of stars are imprinted at the time of stellar formation. Although not the main theme of this IAU Symposium, any conference on the evolution of massive stars would be incomplete without considering the very early formative phases. Of particular interest for predicting the odyssey of an ensemble of massive stars are the initial mass function (IMF) and the initial binary star parameters. While the IMF of massive stars has been a hot topic for a long time (e.g., Lequeux 1979; Hunter 1995; Scalo 1998; Kroupa 2002), the binary properties of massive stars have been more of a side problem, despite several previous spectroscopic studies (see the brief review in Preibisch, Weigelt \& Zinnecker 2001) 
However, an important clue to the origin of high-mass stars has come from the observation that they occur predominantly in binary and multiple systems, apparently more so than low-mass stars (Preibisch, Weigelt \& Zinnecker 2001) and often with very short orbital periods (Mermilliod \& García 2001). Therefore, it seems that the understanding of the formation of high-mass stars is intimately tied to understanding the origin of their binary nature, be it via fragmentation of a massive accretion disk (Yorke \& Sonnhalter 2002), via Bondi-Hoyle accretion onto a lower-mass binary (Maeder \& Behrend 2002), via failed stellar or protostellar collisions (Zinnecker \& Bate 2002) or via dynamical N-body interactions in dense stellar clusters (Bonnell \& Bate 2002).

In this paper, we review the observational situation for unevolved massive binaries on the Main Sequence in young clusters and a number of theoretical ideas regarding their formation. Finally, we discuss some implications and predictions, including a speculation of how the binary properties might change when we consider low metallicity environments.

\section{Generalities and motivation}

\subsection{The definition of massive stars and massive binaries}

We believe there is general agreement about the lower mass limit of a massive star. It is $8 \mathrm{M}_{\odot}$. Why is this a good definition? There are at least three reasons. The first is the fact that stars below $8 \mathrm{M}_{\odot}(\mathrm{B} 3 \mathrm{~V})$ do not emit any Lyman continuum photons. Thus, in terms of spectral type, we define massive stars to

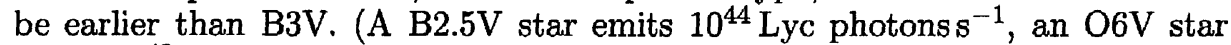
about $10^{49}$.) Secondly, for initial stellar masses above $8 \mathrm{M}_{\odot}$ there is no pre-Main Sequence phase (Palla \& Stahler 1993), i.e., the star ignites H-burning before it finishes accumulating its final mass by accretion from the protostellar gas/dust envelope or circumstellar disk. Thirdly, $8 \mathrm{M}_{\odot}$ is the dividing line between the initial progenitor mass of white dwarfs and type II supernovae. Note that the onset of a significant stellar wind mass loss occurs at around $18 \mathrm{M}_{\odot}(\mathrm{B} 0 \mathrm{~V})$, which is the dividing between O- and B-type stars. Thus, only O-type stars have stellar winds on the Main Sequence which can influence their evolution.

The definition of massive binaries is more tricky. The issue is whether both components must exceed the above $8 \mathrm{M}_{\odot}$ limit, or just the primary of the two. I suggest 'just the primary'. We will use this latter definition here, especially when it comes to the search for physical companions to OB-type stars. Thus a $50+5 \mathrm{M}_{\odot}$ pair or a $10+1 \mathrm{M}_{\odot}$ pair would both be considered as a massive binary according to our definition. Of course, it will be difficult to detect such pairs as close spectroscopic binaries, but we shall see that it is possible to resolve them as visual companions, e.g., in the Orion and M 16 young clusters, using speckle interferometry or adaptive optics.

\subsection{The theme of this contribution}

The underlying theme of this contribution is to study the massive binary properties as a function of the 'environment', in the hope to understand the physics of their formation. For example, comparison of the properties of massive binaries, in young clusters vs. OB associations ought to reveal the role of dynamical gravitational interactions (N-body evolution). These effects are of great importance in dense star clusters, while they should play only a minor part in looser 
aggregates. Another aspect is the origin of runaway OB stars, such as $\zeta$ Pup, O4 I(n)f. Has it been ejected by an asymmetric supernova explosion or has it been released from a young cluster after suffering a tight binary-single star encounter? Finally, we are interested in the initial binary properties as a function of metallicity, particularly in view of the dynamics of the protoglobular clusters in the early universe and in the LMC/SMC. While we will not answer the questions, we want at least to raise them!

As we are concerned with the formation of massive binaries, we will concentrate on observations of young unevolved systems only. The initial or primordial binary properties, as they are sometimes called, consist of the binary frequency, the mass ratios, and the component separations (assuming random orientation of the orbits, the projected separations are nearly equal to the semi-major axes). Measurements of eccentricities are also desirable, as they may hide clues to formation processes (e.g., tidal capture should initially result in large eccentricities for close binaries). One of the key questions is: what is the critical separation or critical period for binary-related mass exchange? I don't know the answer, but perhaps it is of the order of $1 \mathrm{yr}$ for circular orbits and of order $10 \mathrm{yr}$ for highly eccentric orbits. Which fraction of massive binaries (with mass ratio $q>0.1$ ) falls within these limits? My guess is 25-50\%, based on the binary survey of bright O-type stars by Mason et al. (1998).

\section{Review of observations}

\subsection{Definition of the companion star fraction (CSF)}

Many massive stars are actually members of multiple systems, e.g., a hierarchical triple or a Trapezium type quadruple. In order to properly account for these higher order systems, we introduced the term 'companion star fraction' (CSF) defined as $(B+2 T+3 Q) /(S+B+T+Q)$, see Reipurth \& Zinnecker (1993). Here $B$ is the number of binaries (1 companion), $T$ the number of triples (2 companions), and $Q$ is the number of quadruple systems (3 companions). For example: 1 single, 1 binary, 1 triple, and 1 quadruple system in a cluster of four multiple stars like in the Trapezium system $\theta^{1}$ Ori (D, C, B and A) in the center of the Orion Nebula cluster yields CSF $=1.5$ (Preibisch et al. 1999).

This means that a massive star in Orion has on average more than 1 companion, which is significantly different from the case of low-mass pre-Main Sequence stars in Orion, where we typically find CSF $=0.5$ (Prosser et al. 1994; Padgett et al. 1998). This discrepancy is the first hint that the formation process of low-mass and high-mass binary systems may be different.

\subsection{Multiplicity of massive stars in young clusters}

The Orion Nebula Cluster. The multiplicity of the eight massive stars in the Orion Nebula Cluster has been studied by Preibisch et al. (1999), using bispectrum speckle interferometry in the near-infrared $K$-band. These include the four Trapezium stars $\theta^{1}$ Ori A, B, C, and D, $\theta^{2}$ Ori A and B, as well as LPOri and NU Ori. $\theta^{1}$ Ori $\mathrm{C}$, the exciting star of the Orion Nebula has a 33 mas visual companion, previously discovered by Weigelt et al. (1999). Note that $\theta^{1}$ Ori C is a peculiar star, showing strong optical and X-ray variability with a period of 15.4d (Stahl et al. 1996; Babel \& Montmerle 1997), probably related to a $\mathrm{kG}$ magnetic field misaligned with the rotation axis (Donati et al. 2002). It also 
exhibits radial velocity variations, which have been interpreted as a spectroscopic companion which could in fact be identical to the visual companion, if the orbit is sufficiently eccentric (Donati et al. 2002). $\theta^{1}$ Ori A and B have long been known to be spectroscopic binaries (Bossi et al. 1989; Abt et al. 1991), but in addition they also have one and two visual companions, respectively. Simon et al. (1999) even found another infrared companion to $\theta^{1}$ Ori B, so that this system is a Trapezium-type system within the Trapezium. $\theta^{2}$ Ori A is a triple system (spectroscopic binary with a 0'.2 visual companion; Abt et al. 1991, Petr et al. 1998); the same is true for NU Ori. $\theta^{1}$ Ori D and $\theta^{2}$ Ori B are apparently single stars. Adding up the numbers, we find a total of 12 companions, implying $\mathrm{CSF}=12 / 8=1.5$. Interestingly, in all resolved systems the $K$-band flux ratios are quite small, i.e., less than $1 / 3$. This suggests that these visual companions are all significantly less massive than the primary OB stars.

The M16 cluster. Duchêne et al. (2001) have searched for visual binaries among the high mass stars in the M 16 cluster (NGC6611), using adaptive optics. They list some 40 stars of spectral type earlier than B3V that are members of the cluster (age $2 \mathrm{Myr}$, distance $2 \mathrm{kpc}$ ). They find some ten companions (25\%), with separations in the range $0^{\prime \prime} 5$ to $1^{\prime \prime} .5$ (1000-3000 AU), all but two with estimated mass ratios below $q=0.2$. They conclude that these results are consistent with the assumption that companion masses are randomly drawn from the initial mass function. The lack of a strong dependence of the properties of the secondaries on the mass of the primaries lead them to suggest that the canonical picture of cloud fragmentation can explain the binary properties, and there is no need to invoke a different picture for high-mass stars and low-mass stars. This being said, we caution that the low-mass cluster members provide a serious source for false binaries (most companions are physical companions only at the $2 \sigma$ confidence level).

As for spectroscopic binaries, Bosch, Morrell \& Niemela (1999) have studied the radial velocities of the ten earliest type stars in the NGC 6611 cluster. One short-period (W 412, 4d) and two longer period SB2s (W 175, W 197) were found, but three more stars may be variable, among them the most massive object (W 205, O4V).

NGC6231 and other clusters. New statistical results about massive spectroscopic binaries in the young OB cluster were presented by Mermilliod \& García (2001) (see also García \& Mermilliod 2001): (i) the spectroscopic binary frequency among eight clusters rich in O-type stars (i.e., more than five O-type star members) varies from extremely high, of the order of $80 \%$, to rather low, of the order of $15 \%$, seemingly anti-correlated with stellar density in the clusters. NGC 6231, a loose cluster, contains 11 SBs out of 14 stars, while Trumpler 14, the densest cluster in the sample, contains only $1 \mathrm{SB}$ out of 7 O-type stars. An intermediate case is Trumpler 16, with $7 \mathrm{SBs}$ out of 20 O-type star members (35\%); (ii) almost all O-type stars in the 14 young clusters with few O-type stars (i.e., less than three O-type star members) are spectroscopic binaries, often SB2, and even eclipsing. These massive binaries are usually members of hierarchical triple or quadruple systems, or of trapezia, and are often located at the cluster center. $\theta^{1}$ Ori belongs to this category, although $\theta^{1}$ Ori $C$ is not a massive close SB; and (iii) the orbital periods of the spectroscopic binaries 
in the O-type star rich clusters are concentrated in the range $4-5 \mathrm{~d}$, judging mainly from the NGC 6231 and Trumpler 16 clusters; while in the O-type star poor clusters there is an accumulation of orbital periods around $3 \pm 1 \mathrm{~d}$. The orbital eccentricities are high, in the range $0.2-0.6$, at least in NGC 6231 .

These fascinating and surprising facts challenge our views of massive binary formation! In the next section, we take up the challenge, contrasting these clues to some formation models.

\section{Review of theories}

In essence, we can distinguish four types of formation theories:

\subsection{Fragmentation of massive disks around massive stars}

Although direct observational evidence for the presence of circumstellar disks around massive stars is missing (there are indirect hints such as massive outflows, e.g., Beuther et al. 2002; Henning \& Stecklum 2002), let us assume that the disks in question exist, as is known to be the case around low-mass stars. Support for this scenario comes from the 2D rotating collapse calculations by Yorke \& Sonnhalter (2002; see also Yorke 2002). These authors provided simplified numerical simulations of the accretion of a massive protostar for a series of initial cloud cores $\left(30,60,120 \mathrm{M}_{\odot}\right)$ with initially uniform rotation and no magnetic fields. Frequency-dependent radiative transfer was included. Even with rotation, radiation pressure on dust is still a limiting factor for the final mass of the star-disk system. For example, the collapse of a $60 \mathrm{M}_{\odot}$ cloud leads to a $33 \mathrm{M}_{\odot}$ star-disk system, with a substantial fraction (about $1 / 3$ ) still in the disk. Such a massive disk can be gravitationally unstable, provided it is cold enough (formally described by the Toomre criterion). 3D simulations would be needed to follow the evolution (fragmentation) of the disk, but it seems clear that the mass in the disk can only lead to binary systems with mass ratios less than $1 / 3$, as most of the mass goes to the central object. Therefore, disk fragmentation cannot yield nearly equal mass spectroscopic binaries which are so prevalent among the observed massive binaries. Also, disk fragmentation produces only wide binaries, of order 10-100 AU, comparable to the radial disk extent. This mode may then explain the $\theta^{1}$ Ori C companion, but not the SB2 short orbital periods, which correspond to sub-AU semi-major axes.

\subsection{Bondi-Hoyle accretion onto a low-mass protobinary}

Maeder \& Behrend (2002) proposed a model for massive star formation based on a growing accretion rate of the central star, according to $\dot{M} \propto M^{1.5}$. Realising that the model would have to accommodate the high incidence and short periods of massive binaries, they extended their model to include preferential binary formation among massive stars by Bondi-Hoyle accretion from the protocluster gas reservoir. Their idea is as follows. The Bondi-Hoyle accretion rate for an initial binary star (formed by fragmentation or otherwise) is higher than for a single star of the same mass, particularly if the Bondi-Hoyle radius of gravitational influence $\left(b=2 \mathrm{G} M / v^{2}\right.$, where $M$ is the binary mass and $v$ the center-of-mass velocity with respect to the gas) exceeds the semi-major axis of the initial binary system. Then these systems will 'have more of a chance to move to the top of the mass scale and they will do so faster than the other 
single objects. Since mass is accreted on the protobinary system, the semimajor axis will decrease and the orbital periods will also decrease' (Maeder \& Behrend 2002). The problem with this scenario is that the Bondi-Hoyle accretion rate under normal circumstances (gas density $\sim 10^{5} \mathrm{~cm}^{-3}$ and relative velocity $\sim 2 \mathrm{~km} \mathrm{~s}^{-1}$, appropriate for a protocluster cloud) is far too small to supply the required growth of $10^{-5} \mathrm{M}_{\odot} \mathrm{yr}^{-1}$ for solar mass protostars, unless the relative velocity goes to zero. This could happen if the orbital motion of the protobinary partly compensates the relative motion of the gas with respect to the centreof-mass, and each binary component is effectively at rest with respect to the ambient gas, for part of the orbit. Clearly, the situation is very complicated and detailed simulations of binary accretion rates are required ( $c f$. Klessen 2001). Another issue here is, whether the Bondi-Hoyle runaway accretion model can explain the evolution towards equal mass binary components ( $c f$. Bate \& Bonnell 1997).

\subsection{Encounters and tidal capture of intermediate-mass protostars}

This scenario was originally invented to avoid the radiation pressure problem of massive star formation (Bonnell, Bate \& Zinnecker 1998). However, it was soon realised that the collisional build-up of massive stars could also help to form massive almost-touching eccentric binaries, if the kinetic energy of a parabolic encounter could be dissipated as tidal energy ( $c f$. Fabian, Pringle \& Rees 1975) in a gravitationally focussed grazing fly-by (Zinnecker \& Bate 2002). There are several problems with this scenario.

The first one is the extraordinary high stellar density in the center of a cluster that is needed, so that the collisions occur at frequent-enough intervals to be shorter than the stellar evolution time of massive stars (few Myr). Stellar number densities of the order $10^{8}$ stars $\mathrm{pc}^{-3}$ are required, which can only be reached, if a protocluster of intermediate-mass stars undergoes significant contraction and revirialisation owing to accretion onto the stars (see Bonnell et al. 1998). A factor of ten contraction boosts the stellar number density by a factor of 1000 and shortens the collision timescale by a factor of 1000 . This can happen, but the question is: does it happen? No observational support is so far available, although there are hyper-compact $\mathrm{H}$ II regions with dimensions $0.01 \mathrm{pc}$ and a gas number density of more than $10^{6} \mathrm{~cm}^{-3}$ (Kurtz \& Franco 2002). Noone knows what is going on inside those very compact radio sources, as they are so heavily obscured by dust (e.g., IRc2-I in Orion). It is conceivable that cataclysmic events are taking place inside, revealed by explosive outflow phenomena (the famous Orion $\mathrm{H}_{2}$ fingers).

The second problem with this model is the difficulty to first promote and then to avoid stellar mergers. Only in the last step of this runaway process do we need to have a failed stellar merger (i.e., a binary).

The third problem is that the formation of massive binaries seems to require an extreme fine tuning, especially in terms of the impact parameter. Indeed the cross-section for mergers is always larger than that for binary formation. One way out is disk formation during the tidal disruption of the lower-density star in a non head-on collision with a higher-density star (see the example, shown in Zinnecker \& Bate 2002 , of a $3 \mathrm{M}_{\odot}$ pre-MS star disrupted by a $10 \mathrm{M}_{\odot}$ MS-star in a grazing collision). Disk formation increases the cross-section for dissipative capture of the next intruder and may also be a general feature of stellar mergers 
due to the induced rapid stellar rotation (near break-up for realistic non-zero impact parameters). We conclude that tidal capture during near-miss mergers is a viable mechanism to form massive close binaries, but it requires exceptional circumstances.

Finally, there is the issue whether it is pre-Main Sequence stars (perhaps with circumstellar disks) or protostellar cores with extended accretion envelopes that are made to collide (Stahler, Palla \& Ho 2000). These protostellar envelopes offer a much higher interaction cross-section, and provide orbital drag for collisional binary formation (Silk 1978). This variant of the above failed merger picture must be kept in mind, as it works in less extreme and perhaps more realistic conditions.

\subsection{N-body interactions in a young cluster}

Bate \& Bonnell (2002) recently simulated the gas accretion onto 1000 stars in a gas-rich protocluster, using 3D smooth particle hydrodynamics calculations. Accretion forces the cluster to contract, leading to a high-density core where stellar mergers can occur. Small-N groups of massive and intermediate mass stars form due to the self-gravity of the gas, and binary formation in these groups is common, occurring through dynamical three-body capture (different from tidal capture). To begin with, typically a massive star has a lower-mass wide companion. With time, an exchange reaction with a more massive third object occurs, thus forming an almost equal mass but still wide binary. This wide binary then shrinks ('hardens') as it takes up most of the binding energy of the small group being kicked by the other lower-mass group members most of which tend to escape from the group. The typical final outcome is a tight equal mass massive binary, with a lower-mass wide companion (Bate, Bonnell \& Bromm 2002). This picture explains many aspects of massive binaries, including the average companion star fraction $(\mathrm{CSF}=1.5)$ and the prevalence of short-period spectroscopic systems. Also, the timescales for the N-body dynamics are short enough for the whole process to occur on or before the zero age Main Sequence. Therefore, we favour this scenario as our best working hypothesis. There is one nagging doubt, however: one would expect the massive close binary frequency to be correlated with the overall stellar number density in young clusters, which is not what is observed. In fact, the contrary (an anti-correlation) is observed. We have no real explanation at present, how this environmental effect fits into the $\mathrm{N}$-body picture, unless most of the most massive binaries in the densest young clusters have managed to merge into a single object (Zinnecker 1986).

\section{Implications and predictions}

Massive binaries may be the main reason for the existence of many runaway OB stars and field OB stars, expelled from young clusters. The question whether all field O-type stars (10-25\% of all O-type stars) are of some runaway origin is an extremely crucial one; if not, then some O-type stars must be born in isolation rather than in clusters, and many conclusion about massive star formation and indeed massive binary formation must be reconsidered. For example, the collision scenario is clearly in trouble if O-type stars can form outside clusters. As it is, however, we know of at least two examples where B0 stars lie more or less equidistant and in opposite directions of a young cluster (S 255, Zinnecker, 


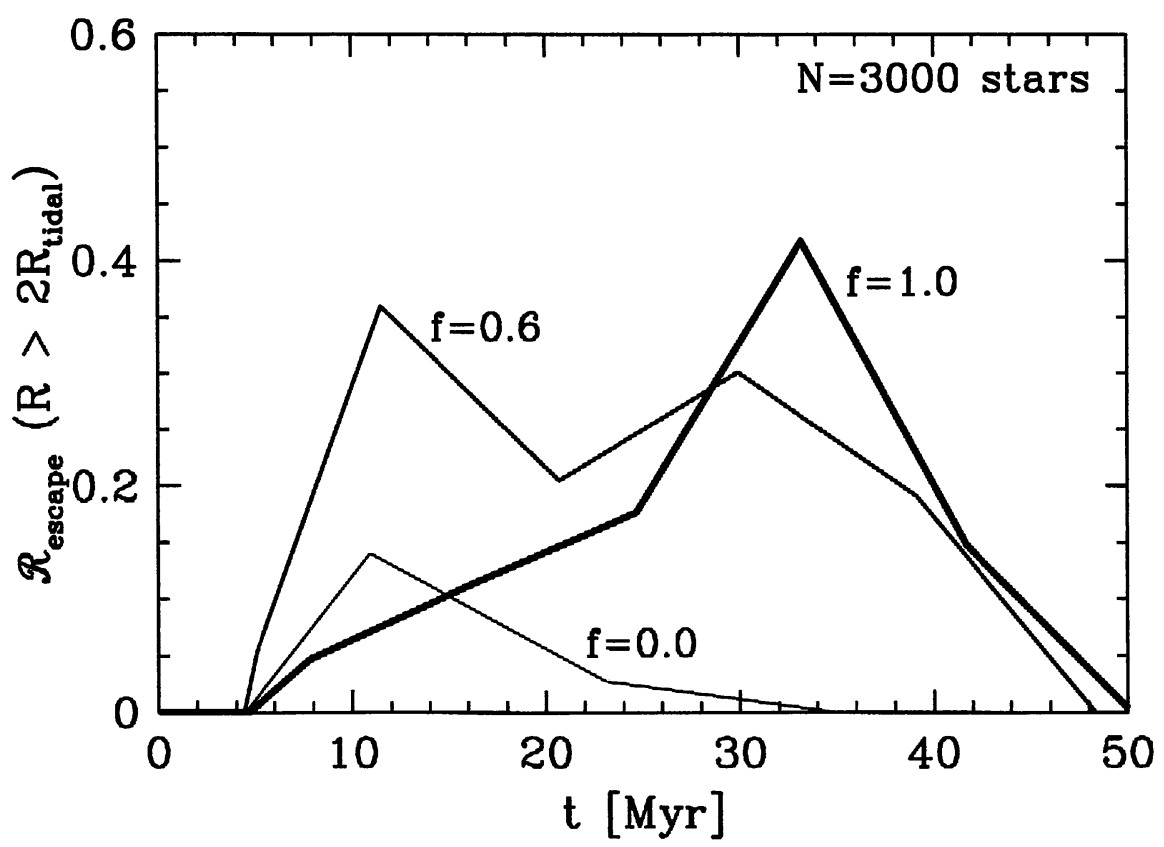

Figure 1. Fraction of massive stars that escape a fully populated cluster of 3000 stars as a function of time after the first dynamical ejection from the cluster. The results of numerical N-body simulations are shown for three different assumptions of the total binary frequency $f$. Random pairing from a field star IMF is assumed to generate the binaries. The fraction of runaway stars (defined to be those stars that reach at least twice the tidal radius of the cluster) is seen to be $20-40 \%$ between $10-40 \mathrm{Myr}$ for realistic binary frequencies. At later times the fraction decreases as the massive stars successively start dying (courtesy of Kroupa 2000).

McCaughrean \& Wilking 1993; Mon R2, Carpenter 2000). The prediction (to be tèsted by observations) is that at least one star in each pair of ejected stars must itself be a binary, to account for the required recoil energy and momentum; see the simulations of Kroupa (2000) who also modelled the fraction of massive stars escaping from a young cluster, thus mimicking an OB field star population (Figure 1).

We end with a speculation on the frequency and orbital separation of massive binaries in lower metallicity star forming regions (e.g., LMC/SMC). We suspect that at lower metallicity the angular momentum problem of star formation is even more severe than for molecular clouds with solar composition, implying that fragmentation must lead to more and/or wider binaries; perhaps even to faster rotation of the binary components. The chain of thought goes as follows: lower metallicity implies fewer heavy elements which are the main donors of free electrons in quasi-neutral clouds. Fewer electrons imply a lesser fractional degree of ionisation. The smaller the degree of cloud ionisation, the shorter is the timescale of retaining a magnetic field during cloud contraction and collapse. Thus the effect of magnetic braking to help solve the angular 
momentum problem (e.g., Mouschovias 1977) is reduced, and the final step of star formation has to get rid of the extra angular momentum by forming wider binary systems in which the remaining excess angular momentum is stored. If this is true, it will have many consequences for low-metallicity populations, but this beyond the scope of this review.

Acknowledgments. I would like to thank Karel A. van der Hucht for his invitation to speak at this Symposium and for his patience with the manuscript. I acknowledge helpful information from Anthony Brown and Pavel Kroupa. I also owe a great deal of thanks to my massive stars co-investigators $M$. Bate, I. Bonnell, Th. Preibisch, G. Weigelt, and also my colleagues A. Maeder, F. Palla, and H.W. Yorke for many stimulating discussions which helped to shape my ideas on massive star formation. Finally, I thank the Brazilian participants of the conference to share with me the experience of the 2002 soccer world-cup final on June 30 (Brazil - Germany 2:0) in the hotel TV room on Lanzarote.

\section{References}

Abt, H.A., Wang, R., Cardona, O. 1991, ApJ 367, 155

Babel, J., Montmerle, T. 1997, ApJ (Letters) 485, L29

Bate, M.R., Bonnell, I.A. 1997, MNRAS 285, 33

Bate, M.R., Bonnell, I.A., Bromm, V. 2002, MNRAS 336, 705

Beuther, H., Schilke, P., Sridharan, T.K., et al. 2002, A\&A 383, 892

Bonnell, I.A., Bate, M.R. 2002, MNRAS 336, 659

Bonnell, I.A., Bate, M.R., Zinnecker, H. 1998, MNRAS 298, 93

Bosch, G.L., Morrell, N.I., Niemela, V.S. 1999, RMxAA 35, 85

Bossi, M., Gaspani, A., Scardia, M., Tadini, M. 1989, A\&A 222, 117

Carpenter, J.M. 2000, AJ 120, 3139

Donati, J.-F., Babel, J., Harries, T.J., et al. 2002, MNRAS 333, 55

Duchêne, G., Simon, T., Eislöffel, J., Bouvier, J. 2001, A\&A 379, 147

Fabian, A.C., Pringle, J.E., Rees, M.J. 1975, MNRAS 172, 15

García, B., Mermilliod, J.-C. 2001, A\&A 368, 122

Henning, Th., Stecklum, B. 2002, in: E.K. Grebel \& W. Brandner (eds.), Modes of Star Formation and the Origin of Field Populations, ASP-CS 285, in press

Hunter, D.A. 1995, RMxAA 3, 1

Klessen, R.S. 2001, ApJ (Letters) 550, L77

Kroupa, P. 2000, in: A. Lancon \& C. Boily (eds.), Massive Stellar Clusters, ASP-CS 211, 233

Kroupa, P. 2002, Science 295, 82

Kurtz, S., Franco, J. 2002, RMxAA-SC 12, 16

Lequeux, J. 1979, A\&A (Letters) 71, L1

Maeder, A., Behrend, R. 2002, in: P.A. Crowther (ed.), Hot Star Workshop III: The Earliest Stages of Massive Star Birth, ASP-CS 267, 179

Mason, B.D., Gies, D.R., Hartkopf, W.I., et al. 1998, AJ 115, 821

Mermilliod, J.-C., García, B. 2001, in: H. Zinnecker \& R.D. Mathieu (eds.), The Formation of Binary Stars, Proc. IAU Symp. No. 200 (San Francisco: ASP), p. 191

Mouschovias, T.Ch. 1977, ApJ 211, 147

Padgett, D.L., Strom, S.E., Ghez, A. 1997, ApJ 477, 705 
Palla, F., Stahler, S.W. 1993, ApJ 418, 414

Petr, M.G., Coude Du Foresto, V., Beckwith, St.V.W., et al. 1998, ApJ 500, 825

Preibisch, Th., Balega, Y., Hofmann, K.-H., et al. 1999, New Astron. 4, 531

Preibisch, Th., Weigelt, G., Zinnecker, H. 2001, in: H. Zinnecker \& R.D. Mathieu (eds.), The Formation of Binary Stars, Proc. IAU Symp. No. 200 (San Francisco: ASP), p. 69

Prosser, Ch.F., Stauffer, J.R., Hartmann, L., et al. 1994, ApJ 421, 517

Reipurth, B., Zinnecker, H. 1993, A\&A 278, 81

Scalo, J. 1998, in: G. Gilmore \& D. Howell (eds.), The Stellar Initial Mass Function, ASP-CS 142, 201

Silk, J. 1978, in: T. Gehrels (ed.), Protostars and Planets. I (Tucson: Univ. of Arizona Press), p. 187

Simon, M., Close, L., Beck, T. 1999, AJ 117, 1375

Stahl, O., Kaufer, A., Rivinius, T., et al. 1996, A\&A 312, 539

Van Bever, J., Vanbeveren, D. 1998, A\&A 334, 21

Wang, Q.D., Gotthelf, E.V., Lang, C.C. 2002, Nature 415, 148

Weigelt, G., Balega, Y., Preibisch. Th., et al. 1999, A\&A (Letters) 347, L15

Yorke, H.W. 2002, P.A. Crowther (ed.), Hot Star Workshop III: The Earliest Stages of Massive Star Birth, ASP-CS 267, 165

Yorke, H.W., Sonnhalter, C. 2002, ApJ 569, 846

Zinnecker, H. 1986, in: C.W.H. de Loore, A.J. Willis \& P. Laskarides (eds.), Luminous Stars and Associations in Galaxies, Proc. IAU Symp. No. 116 (Dordrecht: Reidel), p. 271

Zinnecker, H., McCaughrean, M.J., Wilking, B.M. 1993, in: E.H. Levy \& J.I. Lunine (eds.), Protostars and Planets. III (Tucson: Univ. of Arizona Press), p. 429

Zinnecker, H., Bate, M.R. 2002, P.A. Crowther (ed.), Hot Star Workshop III: The Earliest Stages of Massive Star Birth, ASP-CS 267, 209

\section{Discussion}

WILLIAMS: What is the influence of selection effects on the incidence of companions as a function of type?

ZINNECKER: At present we don't know if there are close binaries with extreme mass ratios (not detectable as spectroscopic binaries) and we don't know at all which sort of companions exist in the range of intermediate separations, too close to be seen as visual binaries in high-spatial resolution observations and too wide to show up as spectroscopic binaries. This gap in our knowledge may be bridged to some extent by future interferometric studies reaching a spatial resolution of the order of 1 mas (1 AU at $1 \mathrm{kpc}$ distance) and brightness ratios 1:100. In summary, I believe that the census of massive stars with lower mass companions is still quite incomplete.

FoELLMI: There is an impressive number of scenarios of interacting massive binaries. We have undertaken in Montreal a systematic search for Wolf-Rayet binaries in the Magellanic Clouds. We found: $\sim 40 \%$ binary frequency in the SMC, $15-25 \%$ in the LMC, no evidence for accretion in the secondaries, and intrinsic hydrogen in the wind of most of the (single and binary) WR stars. This argues against binary evolution for WR stars, where it is theoretically the most expected (i.e., in a low- $Z$ environment).

ZINNECKER: Your new data are very important constraints for stellar evolution and the close binary frequency of massive stars. However, they do not constrain the fre- 
quency of wide binaries. In my theoretical speculation, a lower- $Z$ environment tends to favour wider separations of binaries and likely faster rotation of the components, i.e., in my view a lower- $Z$ environment produces a higher angular momentum population of massive stars. As an aside, I never claimed that WR stars originate through the binary channel. In fact, this old concept probably was invented at a time when we had no idea about the importance of winds from massive stars. (Even at SMC/LMC metallicities, stellar winds can do the trick to form WR stars, without a binary channel).

LEITHERER: I do not see why I would make a large error in the interpretation of data of unresolved starburst populations if I assumed all stars were single. Unless stellar evolution changes (which may indeed be the case) very little should change.

ZINNECKER: It all depends on the frequency of interacting close massive binaries which can lead to stellar mass exchange and even stellar mergers for the closest systems. Consequently, some rejuvenation of a fraction of the massive stars can occur, as pointed out by Van Bever and Vanbeveren (1998), implying that binary evolution can make a starburst look younger than it really is, say $5 \mathrm{Myr}$ instead of $10 \mathrm{Myr}$. A high binary frequency can make a difference here.

CherepashChuK: Ignoring binaries in starbursts will make a big difference for the presence of compact objects in post-starbursts, and the associated accretion effects (X-ray binaries).

ZINNECKER: Indeed, thank you, Mr. chairman. (HZ shows the Chandra X-ray image of the Galactic Centre region with some 1000 point sources; from Wang et al. 2002.)

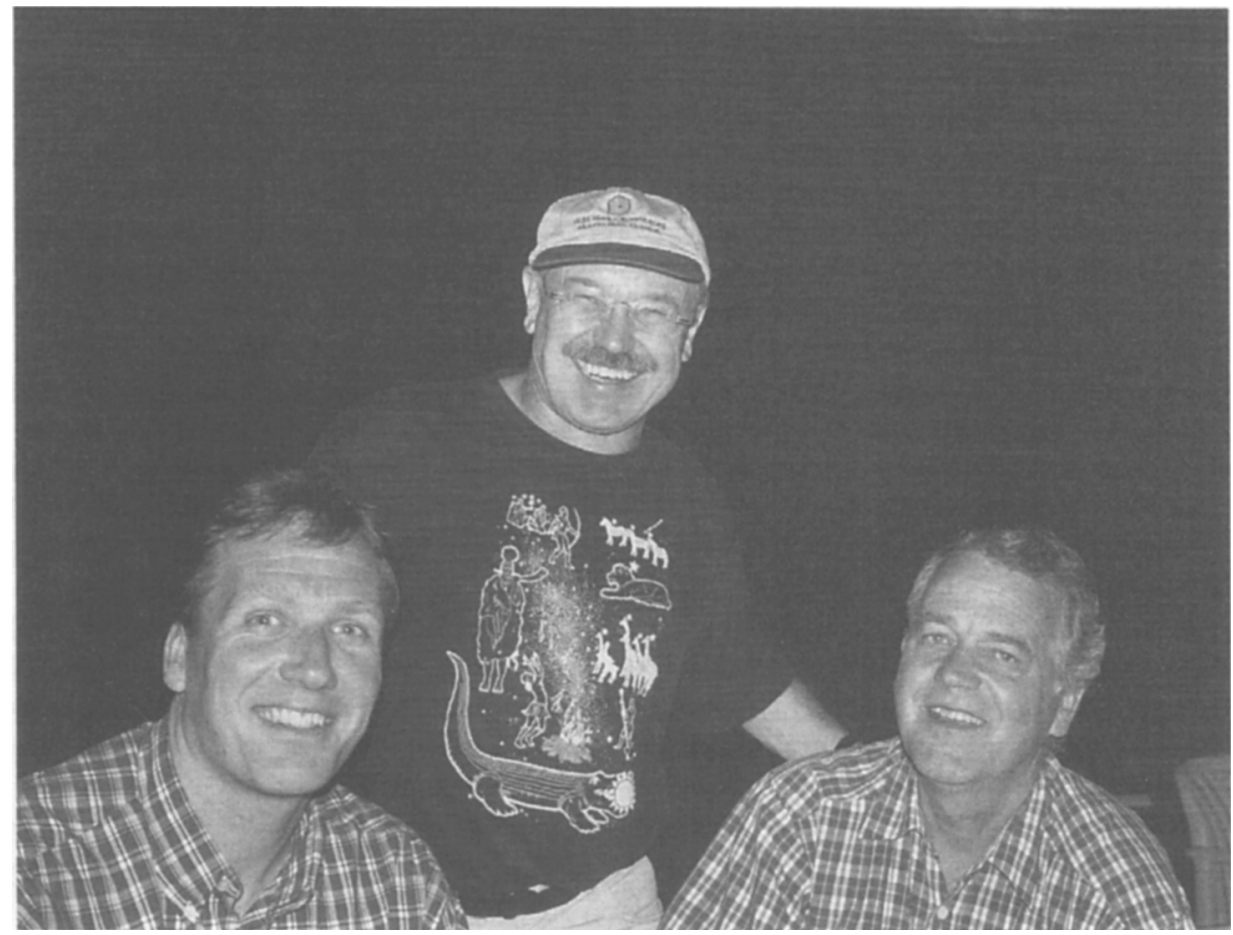

No strangers in the night: Lex Kaper, Hans Zinnecker, and Tony Moffat 